Spaliviero, C. (2018). Didattica della grammatica italiana in prospettiva LS: una risorsa didattica. Italica Wratislaviensia, 9(2), 255-275.

DOI: http://dx.doi.org/10.15804/IW.2018.09.26

Camilla Spaliviero

Università Ca' Foscari Venezia

\title{
DIDATTICA DELLA GRAMMATICA ITALIANA IN PROSPETTIVA LS: UNA RISORSA DIDATTICA
}

\author{
TEACHING ITALIAN GRAMMAR \\ AS A FOREIGN LANGUAGE: A DIDACTIC RESOURCE
}

\begin{abstract}
Teaching Italian grammar involves several critical aspects, amongst which is a recurring lack of motivation in students. Therefore, combining the teaching of grammar with the use of potentially appealing resources for students is a challenge for the teacher of Italian as a foreign language (FL). With regards to this, songs represent an important didactic resource; in fact, they can motivate students' learning, are texts full of linguistic and cultural references (such as the variety of sociolinguistic registers or rhetorical figures), and foster the memorisation of lexical and grammatical structures thanks to their intrinsic characteristics (like rhythm and melody). In order to take advantage of the potentialities of songs, the teacher of Italian as a FL should acquire both appropriate methodological competences and a broad collection of didactic techniques to present the various grammatical topics.

The essay is divided into four sections: the first attempts to explore the teaching of Italian grammar from a humanistic perspective; the second is devoted to the presentation of the critical aspects that characterise it, together with the introduction of some possible solutions, such as the use of songs, which is debated in the third section. Finally, the fourth and last part is concerned with some didactic considerations about how and when to use songs for grammatical purposes.
\end{abstract}

Keywords: language education, grammar, Italian as a FL, didactic resource, song 


\section{DIDATTICA DELLA GRAMMATICA ITALIANA LS NELLA PROSPETTIVA UMANISTICA}

el corso del Novecento, le teorie e i modelli di riferimento didattici
relativi all'insegnamento della grammatica sono stati oggetto di diverse trasformazioni, parallelamente all'evoluzione della più ampia disciplina della glottodidattica. Il rinnovamento delle idee riguardanti che cosa significhi sapere una lingua hanno avuto, infatti, ripercussioni sulle definizioni, sulle funzioni e sulle modalità di insegnamento grammaticale. Per quanto attiene all'educazione linguistica, alle mete educative generali di "culturizzazione", "socializzazione" e "autopromozione"1 (Balboni, 2011, pp. 88-89), si associa l'acquisizione della competenza comunicativa (cfr. Hymes, 1972). Per ragioni di sintesi del nostro contributo, ci limitiamo a descrivere brevemente il modello di competenza comunicativa di Paolo E. Balboni (2014, p. 86), illustrato nella FIGURA 1.

Secondo Balboni, la competenza comunicativa è "una realtà mentale che si realizza nel mondo, in eventi comunicativi realizzati in contesti sociali dove si usa l'italiano per compiere un'azione, per raggiungere uno scopo" (ibidem). Per sviluppare la capacità di "saper fare con la lingua", ovvero di riuscire ad agire efficacemente all'interno dei differenti contesti comunicativi rispettando le regole sociali, pragmatiche e culturali che li caratterizzano, è necessario "saper fare lingua", vale a dire poter tradurre le competenze mentali in azioni comunicative grazie alla padronanza delle abilità linguistiche (primarie e integrate) che permettono di comprendere, produrre e manipolare diverse tipologie di testi.

1 La "culturizzazione" corrisponde alla relazione che ogni individuo stabilisce con gli 'altri', ovvero i gruppi linguistico-culturali che popolano il mondo in cui si vive; si identifica con l'inculturazione nella propria cultura materna e con l'acculturazione negli ambienti culturali non nativi per diventare componente integrante dei suddetti gruppi. La "socializzazione" è fondata sul rapporto tra l'individuo e i diversi 'tu' con cui è possibile interagire; una volta portato a termine il processo di culturizzazione, si possono stabilire delle relazioni significative e portare a termine i propri obiettivi umani, interagendo efficacemente nella società in cui si vive. L'“autopromozione" si basa sulla relazione che ciascun individuo stabilisce con se stesso, al fine di realizzare il proprio progetto esistenziale. 
Presupposto ulteriore è la capacità di "sapere la lingua", ovvero possedere tre diversi tipi di competenze mentali corrispondenti ad altrettanti sistemi di regole: le competenze linguistiche (fonologiche, morfosintattiche, testuali e lessicali), le competenze extralinguistiche (cinesiche, prossemiche, oggettemiche e vestemiche) e le competenze contestuali (sociolinguistiche, pragmalinguistiche e interculturali).

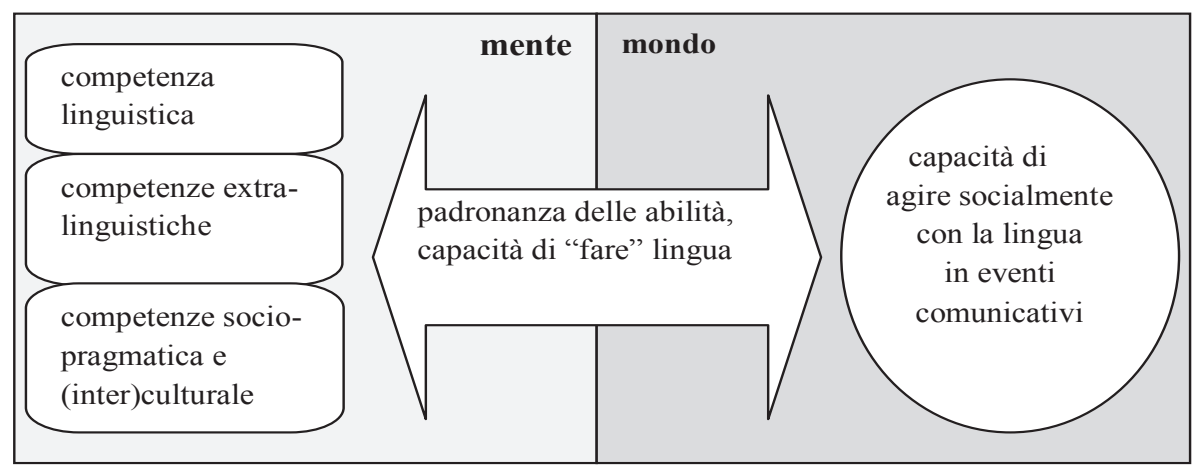

FIGURA 1: Il modello di competenza comunicativa di P.E. Balboni

Per ciò che riguarda nello specifico la didattica della grammatica, dal modello di competenza comunicativa si può evincere che la competenza linguistica, composta da differenti tipi di 'grammatiche' come sopra indicato, rappresenta 'solo' uno degli elementi che costituiscono l'educazione linguistica e non più il fine esclusivo o principale dell'insegnamento della lingua. All'interno di una prospettiva glottodidattica di matrice umanistica risultano fondamentali (Caon, 2008):

a. la considerazione della lingua come mezzo per agire socialmente;

b. il riconoscimento della centralità dei bisogni e degli interessi dello studente;

c. il ruolo del docente come facilitatore dell'apprendimento linguistico;

d. i principi di 'bimodalità' e 'direzionalità' che regolano il funzionamento congiunto ma differente dei due emisferi celebrali (qui approfonditi nel paragrafo 4);

e. la centralità della componente emozionale nel processo di apprendimento linguistico (qui approfondita nel paragrafo 2); 
f. il rispetto delle sequenze naturali di acquisizione linguistica;

g. il sostegno di modalità di lavoro tra pari e cooperative mediante le metodologie a 'mediazione sociale'.

Per quanto concerne specificamente la dimensione linguisticogrammaticale, la glottodidattica di natura umanistica è caratterizzata, inoltre, dal superamento degli approcci:

a. formalistici, di impostazione grammatico-traduttiva e basati sullo studio delle forme grammaticali della lingua mediante modalità deduttive ed eterodirette;

b. strutturalistici, fondati sulla memorizzazione delle strutture grammaticali attraverso lo sviluppo di meccanismi automatici impostati sul binomio stimolo-risposta e il successivo rinforzo.

Ad essi si preferisce sempre più una modalità induttiva orientata alla scoperta delle regole morfosintattiche, o comunque una didattica della grammatica che integri le modalità induttiva e deduttiva in un'ottica di equilibrio tra induzione e deduzione, soprattutto nel caso dell'italiano LS, con il progressivo affermarsi del concetto di 'riflessione sulla lingua' rispetto alla precedente idea di 'insegnamento grammaticale'.

Come scrive Maria Cecilia Luise (2014), entrambi i tipi di processi presentano dei vantaggi e degli svantaggi. Se la grammatica deduttiva rischia di essere percepita come noiosa da parte degli studenti che svolgono un ruolo più passivo, è innegabilmente più economica in termini di tempo e più facile da gestire da parte dell'insegnante - aspetto non trascurabile nella prospettiva della didattica dell'italiano LS, concentrata generalmente in poche ore settimanali e basata quasi totalmente sull'input controllato da parte del docente. Viceversa, la grammatica induttiva

2 Il procedimento deduttivo, tipico dell'insegnamento grammaticale tradizionale, è 'esplicito' in quanto va dal generale al particolare: l'insegnante presenta le regole morfosintattiche allo studente all'interno di schemi pieni chiedendogli di memorizzarle e di applicarle in una serie di esercizi per verificarne l'applicabilità e le eventuali eccezioni. Il procedimento induttivo, invece, sostenuto dagli approcci glottodidattici più recenti, è 'implicito' poiché procede dal particolare al generale: sotto la guida dell'insegnante, lo studente è portato a riempire degli schemi vuoti o semi-vuoti attraverso l'osservazione delle strutture morfosintattiche all'interno del testo, a scoprire le regole e le eventuali eccezioni e a formulare delle ipotesi sul loro funzionamento linguistico. 
è più dispendiosa in termini di tempo e maggiormente complessa da coordinare da parte dell'insegnante ma è anche potenzialmente più motivante per gli studenti e favorisce il raggiungimento della meta educativa dell' 'autopromozione'. Infatti, grazie agli schemi aperti, costantemente integrabili a mano a mano che si procede con l'osservazione, i discenti possono percepire il piacere di esseri coinvolti attivamente nella costruzione della propria grammatica personale, memorizzare più facilmente le strutture morfosintattiche individuate e allenare la propria autonomia nell'apprendimento linguistico.

Realisticamente, allora, si riconosce la necessità di strutturare la didattica della grammatica attraverso l'integrazione di entrambe le procedure, impiegate in modi e momenti differenti. Le ragioni di tale 'compromesso' non rimandano solo al fattore tempo, ma riguardano anche la natura della lingua italiana. Come scrive Balboni (2014, p. 101),

[...] ogni acquisizione parte da una grammatica implicita, ma è poi necessaria una focalizzazione esplicita sulle grammatiche, soprattutto per lingue romanze in cui la morfologia è estremamente ricca. Recenti ricerche [...] e sperimentazioni $[. .$.$] hanno dimostrato come una focalizzazione esplicita$ su alcuni elementi grammaticali possa farli risaltare nella grande massa di forme che compaiono nell'input e quindi possa facilitarne l'acquisizione.

Pertanto, è consigliabile adottare quella che Marco Mezzadri definisce una "posizione eclettica", in cui "la grammatica ricopre ruoli e richiama l'attenzione didattica in modo diverso a seconda delle fasi della lezione, senza pregiudizi né preclusioni" (2015, p. 231). Il carattere duttile di tale atteggiamento non esclude l'esigenza di osservare precise coordinate metodologiche: "ciò che occorre coscientemente applicare è la corretta sequenza delle fasi che porta a far precedere la globalità all'analisi, l'acquisizione all'apprendimento, la lingua alla metalingua, il significato alla forma" (ivi, p. 236).

All'interno di un curricolo linguistico, ne consegue che:

a. non si possono inserire tutte le 'grammatiche' che definiscono la competenza linguistica e si devono presentare gli aspetti basilari di quelle presenti; 
b. è necessario rispettare l'ordine naturale di acquisizione linguistica, i ritmi e le modalità di apprendimento dei discenti, seguendo un procedimento a spirale (che riprende quanto già affrontato in precedenza, integrandolo con nuove informazioni);

c. lo studio della lingua e delle diverse 'grammatiche' che la costituiscono è al servizio dello studente e mira allo sviluppo della capacità di comprendere e produrre messaggi linguistici, oltre all'acquisizione della competenza metalinguistica (Balboni, 2012).

L'insegnamento delle lingue (e, quindi, anche dell'italiano LS) mira all'acquisizione di due tipi di competenze differenti e complementari: la 'competenza d'uso', ovvero lo sviluppo della dimensione funzionale che prevede l'impiego della lingua a fini comunicativi, e la 'competenza sull'uso', vale a dire il potenziamento della dimensione metacognitiva con la maturazione delle capacità di analisi, di classificazione e di riflessione linguistica, che consente il passaggio dalla capacità di usare le regole per comunicare alla capacità di descrivere e ragionare esplicitamente sulle regole stesse.

L'acquisizione della competenza metacognitiva può incidere positivamente sul potenziamento delle realizzazioni linguistiche degli studenti ma è principalmente finalizzata a insegnare a ragionare (nell'ottica del docente) e a imparare ad imparare per diventare sempre più autonomi (nella prospettiva dello studente), con la sovraestensione delle suddette competenze ad altre discipline e alla realtà extrascolastica.

\section{CRITICITÀ E POSSIBILI SOLUZIONI LEGATE ALLA DIDATTICA DELLA GRAMMATICA}

La rinnovata definizione delle funzioni e delle modalità di insegnamento delle strutture morfosintattiche all'interno della glottodidattica di matrice umanistica ribadisce l'essenzialità della didattica della grammatica (seppur con le specificità menzionate nel paragrafo precedente) all'interno del più ampio curricolo di italiano LS.

Questo implica, a livello teorico (paragrafo 1) e operativo (paragrafi 3 e 4), che gli obiettivi dell'insegnamento linguistico siano l'acquisizione della competenza sia comunicativa sia metacomunicativa e che la 
riflessione linguistica debba prevedere un coinvolgimento attivo dello studente all'interno di situazioni comunicative motivanti, significative e possibilmente autentiche.

L'affermazione della glottodidattica di natura umanistica, tuttavia, non comporta la risoluzione delle tradizionali criticità che contraddistinguono questo ambito di studi. La difficoltà principale dell'insegnamento grammaticale si deve alla natura astratta della suddetta disciplina. A differenza, ad esempio, dei contenuti lessicali che appaiono più facili da collegare all'esperienza concreta del mondo e, dunque, all'interno di mappe o reti semantiche, i contenuti grammaticali sono spesso percepiti come difficili, poco piacevoli e demotivanti da parte degli studenti poiché lontani dalla realtà concreta nella quale essi sono immersi quotidianamente.

Nelle diverse opinioni degli studenti di italiano LS relative allo studio della grammatica raccolte da Cecilia Andorno, Franca Bosc e Paola Ribotta (2003), gli aggettivi che si ripetono con più frequenza sono 'noiosa', 'seria' e 'faticosa'. Rispetto alle principali difficoltà che gli insegnanti di italiano LS riscontrano nella didattica della grammatica, dai questionari di Günter Zimmermann (1990) emerge che l'aspetto più problematico corrisponde alla presentazione delle regole grammaticali attraverso delle modalità motivanti e interessanti per i discenti.

Uno dei punti chiave per risolvere tale situazione critica risiede proprio nella motivazione, senza la quale non si creano le condizioni per l'acquisizione stabile e duratura della lingua. Per essere motivato allo studio della grammatica, lo studente deve emozionarsi, percependo il piacere, tra gli altri, di 'apprendere' (grazie alla proposta di compiti fattibili e alla considerazione degli errori come tappa naturale all'interno del processo di acquisizione), della 'sfida' e della 'sistematizzazione' (mediante attività stimolanti che richiedono di scoprire i meccanismi di funzionamento linguistico-grammaticale; cfr. Balboni, 2012).

Nella tradizione glottodidattica italiana, Renzo Titone (1987) è uno dei primi a considerare il ruolo dell'emozione e a riconoscere il ruolo centrale della motivazione - 'elemento dinamogetico' dell'apprendimento - all'interno del processo di acquisizione linguistica. Secondo il suo 'modello egodinamico', ogni studente elabora delle strategie per 
realizzare il progetto di sé, corrispondenti nel nostro caso all'acquisizione della grammatica italiana in un contesto LS. Il 'momento tattico' si verifica durante il contatto reale tra il progetto e il risultato: se il feedback è positivo, e quindi le strategie risultano valide, l'ego continua a dare energia, sostenendo la motivazione anche rispetto a compiti non piacevoli ma ritenuti comunque necessari per la realizzazione dell'obiettivo.

Successivamente, facendo riferimento alla 'teoria cognitiva delle emozioni’ elaborata da Magda B. Arnold negli anni Sessanta, John H. Schumann (1997) sostiene l'importanza di puntare sulla motivazione 'intrinseca' dello studente (non legata a fattori esterni, eterodiretti, ma basata invece su ragioni personali, autodirette), poiché i processi cognitivi si generano solo in seguito allo sviluppo dei processi emotivi ${ }^{3}$.

Risulta fondamentale, quindi, cercare una mediazione tra l'obbligatorietà di affrontare dei contenuti che, come quelli grammaticali, sono di frequente distanti dagli interessi e dalle necessità istintivamente percepite degli studenti - e quindi potenzialmente poco stimolanti e l'opportunità di acquisire gli stessi argomenti attraverso delle esperienze motivanti che soddisfino i bisogni e incontrino le preferenze dei discenti ${ }^{4}$.

Il ruolo chiave per lo sviluppo della motivazione intrinseca rispetto all'acquisizione grammaticale è svolto dal docente che compie delle scelte didattiche precise e consapevoli riguardanti:

a. i contenuti, se soddisfano e assecondano naturalmente i bisogni e le inclinazioni degli studenti;

b. le metodologie, che possono rendere più facile e piacevole lo studio di contenuti di per sé poco motivanti per i discenti;

c. le risorse, che si possono collegare a esperienze precedenti e ai gusti degli apprendenti;

3 Per approfondire il ruolo delle emozioni nell'acquisizione linguistica si veda Balboni, 2013.

4 Per un'esaustiva presentazione dell'evoluzione delle teorie sulla motivazione, in particolare nella tradizione metodologica veneziana, si veda Caon, 2006. 
d. la relazione, sia con il docente (nel ruolo professionale di guida e umano di persona) sia con i compagni, costruita sui valori di trasparenza, ascolto reciproco, fiducia e rispetto (Caon, 2011);

e. i materiali e gli ambienti didattici (fisici e virtuali, come internet), che influenzano sia la motivazione sia la qualità dell'acquisizione (Caon \& Serragiotto, 2012).

Nel presente contributo, ci focalizziamo sulla proposta di utilizzare la risorsa della canzone per la didattica della grammatica italiana in prospettiva LS, esplicitandone alcuni aspetti metodologici.

\section{UNA RISORSA PER LA DIDATTICA DELLA GRAMMATICA: LA CANZONE}

In termini generali, la canzone può rappresentare un valido strumento per la didattica dell'italiano in prospettiva LS. Dal punto di vista glottodidattico, è un materiale che David A. Wilkins definisce 'autentico' (1976), poiché originariamente creato per un pubblico di madrelingua e non per studenti stranieri a scopi didattici. Questa natura implica una non gradualità dei testi, che si presentano, così, stratificati su differenti livelli, compromettendone la comprensibilità in assenza di un attento lavoro di selezione e di adattamento da parte del docente.

L'uso della canzone a fini didattici non è, infatti, esente da possibili criticità, tra cui ricordiamo:

a. a livello contenutistico: la presenza di una grande varietà di registri linguistici, di livelli stilistici differenti e di impliciti culturali a discapito della comprensione, oltre all'uso letterario della lingua di difficile accessibilità per i livelli iniziali;

b. a livello interpretativo: la pronuncia poco chiara da parte degli interpreti e la disparità tra il ritmo del cantato e il ritmo del parlato quotidiano (isoritmia);

c. a livello motivazionale: le perplessità da parte degli studenti, che possono non amare la musica o considerare pregiudizialmente poco utile e 'serio' l'uso della canzone nella classe di lingua;

d. a livello organizzativo: la necessità di predisporre lo spazio d'aula con adeguati dispositivi tecnici. 
Nella nostra prospettiva di una glottodidattica di natura umanistica, tuttavia, l'uso della canzone in quanto materiale 'autentico' è di rilevante interesse, poiché offre molteplici spunti linguistici (oltre che culturali e letterari) e può favorire l'acquisizione di strutture morfosintattiche grazie alle peculiarità che la caratterizzano.

Le potenzialità dell'impiego didattico della canzone sono numerose e si identificano con:

a. il fattore motivazionale;

b. la maggiore facilità di memorizzazione dei contenuti lessicali e grammaticali grazie alle sue caratteristiche intrinseche (come il ritmo e la melodia; cfr. Caon, 2011);

c. l'opportunità di focalizzarsi su uno o più elementi linguistici di tipo sociolinguistico, morfosintattico e fonetico;

d. l'occasione di approfondire degli aspetti culturali attraverso lo studio, ad esempio, di modi di dire, eventi storici, espressioni dialettali, anche in un'ottica interculturale;

e. la possibilità di riflettere sulla ricercatezza formale dei termini a livello letterario.

In particolare, utilizzare la canzone in chiave didattica può presentare notevoli vantaggi motivazionali, fondamentali per poter attivare la curiosità degli studenti e favorire l'acquisizione stabile e duratura della lingua, poiché appartiene alla quotidianità degli apprendenti di diverse età, può trattare temi a loro vicini e si può proporre attraverso metodologie ludiche e cooperative che favoriscono la partecipazione attiva dei discenti.

Il piacere relazionato all'ascolto musicale, infatti, può incidere positivamente sull'acquisizione. Come sostiene Franco Fabbro, "le strutture emotive del sistema nervoso sono fortemente coinvolte nei processi di fissazione dei ricordi nella memoria", di conseguenza "le situazioni che coinvolgono il sistema emozionale" permettono agli studenti di voler "ripetere ciò che è piacevole" (1996, p. 110). Inoltre, le componenti musicali e linguistiche che caratterizzano i testi delle canzoni consentono il coinvolgimento di entrambi gli emisferi cerebrali, stimolando quello destro dal punto di vista emotivo e quello sinistro a livello razionale, con conseguenti benefici a livello glottodidattico. A questo proposito, 
Mario Cardona scrive che "così come esiste un ritmo nella musica esiste anche un ritmo nelle lingue e forse il saper cogliere lo specifico ritmo di una determinata lingua, così come la sua intonazione, rappresenta una competenza che può favorire l'apprendimento linguistico" (2009, pp. 7-8). L'integrazione di stimoli affettivi e cognitivi attraverso l'uso della canzone può, così, favorire la memorizzazione delle strutture morfosintattiche.

Posto che la ripetizione è la caratteristica principale dell'acquisizione linguistica, essa ne rappresenta, al contempo, il problema principale poiché può produrre noia. L'ascolto reiterato della canzone, invece, può non annoiare per le potenzialità summenzionate ma risultare anzi piacevole, permettendo il verificarsi del 'rule of forgetting' teorizzato da Stephen Krashen (1983), per cui si progredisce nell'acquisizione linguistica quando ci si dimentica di stare imparando.

Inoltre, l'inserimento dei contenuti morfosintattici all'interno di un testo strutturato dal punto di vista narrativo come la canzone permette di eludere la natura astratta, isolata e decontestualizzata della grammatica (Falioni, 1993) a cui ci siamo riferiti precedentemente (paragrafo 2).

È necessario precisare che, inizialmente, l'ascolto ripetuto delle canzoni può agevolare la memorizzazione delle strutture morfosintattiche solo a un primo livello 'superficiale', perché non grammaticalizzato. Rita Pasqui afferma a tal proposito (cito come in Caon, 2011, p. 23):

Capita anche che gli studenti, ascoltando al di fuori del contesto scolastico delle canzoni in L2/LS che amino particolarmente, ricordino alcune parole (dinning in their heads, che "frullano" nella loro mente) pur senza conoscerne il significato: la canzone funzionerebbe pertanto come un attivatore involontario del LAD che trasforma l'input (non solo l'input comprensibile) in intake.

Questo fenomeno è spesso associato alla ripetizione insistita delle canzoni definite 'tormentoni', di cui si ricordano inconsapevolmente sia la melodia sia le parole anche se a un primo ascolto possono non risultare piacevoli. È l'azione successiva del docente a permettere di sensibilizzare e di rendere consapevoli gli studenti rispetto ai contenuti grammaticali presenti nel testo della canzone, che in questo modo sono 
formalizzati. L'insegnante può decidere se affrontare le strutture morfosintattiche prestabilite prima delle attività sulla canzone, lasciando a quest'ultima il compito di fissare le conoscenze grammaticali, o dopo, guidando gli studenti verso la loro scoperta e la loro sistematizzazione a posteriori.

Facendo riferimento al contesto LS, la selezione dei contenuti grammaticali da proporre agli studenti attraverso le canzoni è particolarmente rilevante. Infatti, da un lato, la maggior parte dell'input linguistico che giunge ai discenti è proposto, coordinato e controllato interamente dal docente (Santipolo, 2006). Dall'altro, trattandosi di un testo non graduato né stratificato, è fondamentale proporre delle canzoni in cui le strutture morfosintattiche che si desiderano affrontare in classe emergano significativamente.

Inoltre, per predisporre positivamente gli studenti rispetto ai contenuti morfosintattici e sostenere il loro coinvolgimento nel corso delle attività didattiche, è essenziale che la metodologia proposta dal docente per utilizzare la canzone a fini grammaticali sia diversificata. Una delle strategie che il docente può attuare per fare ritornare i discenti sulle medesime strutture senza demotivarli è affrontare gli stessi contenuti attraverso modalità differenti riguardanti: lo svolgimento delle attività, le tipologie di tecniche glottodidattiche e le risorse didattiche.

Il fine ultimo di impiegare la canzone per la didattica dell'italiano a studenti stranieri è, dunque, quello di "attivare il soggetto partendo dall'analisi dei suoi bisogni formativi e dei suoi interessi, per giungere attraverso un patto formativo - alla mediazione tra aspirazioni e desideri personali e necessità dell'ambiente in cui si opera" (Caon, 2011, p. 20). L'insegnante, da un lato, si propone di facilitare l'apprendimento linguistico attraverso strumenti potenzialmente piacevoli per i discenti come la canzone e, dall'altro, deve essere capace di adattare la risorsa agli obiettivi didattici del corso e al livello degli studenti. Tale incontro permette, così, la creazione di un 'ponte' tra le esigenze della realtà scolastica tradizionale e le inclinazioni sviluppate dagli studenti nel contesto extrascolastico.

Affinché le potenzialità della canzone siano realmente effettive, è quindi fondamentale il ruolo di facilitatore dell'insegnante. Visti i pro e i contro 
legati all'uso didattico della canzone, è evidente come non sia il mezzo bensì chi lo usa a determinarne la qualità. A livello operativo, coerentemente con una glottodidattica di natura umanistica (paragrafo 1), l'insegnante è chiamato a (Caon, 2011):

a. selezionare le canzoni in base al livello linguistico dei discenti e alle loro preferenze musicali;

b. creare delle attività motivanti e sfidanti dal punto di vista cognitivo;

c. condividere e negoziare le ragioni a sostegno dell'impiego della canzone;

d. proporre delle attività mirate allo sviluppo delle competenze sia cognitive sia sociali, attraverso metodologie cooperative e ludiche, senza dimenticare di introdurre esercizi più liberi, espressivi e creativi;

e. predisporre le attività con la canzone in un luogo adatto dal punto di vista tecnico-sonoro e senza dilatarne eccessivamente i tempi.

\section{CONSIDERAZIONI METODOLOGICHE PER L'UTILIZZO DIDATTICO DELLA CANZONE A FINI GRAMMATICALI}

I due principali argomenti di discussione relativi alla didattica della grammatica riguardano, da un lato, l'opposizione tra 'insegnamento grammaticale' e 'riflessione sulla lingua' e, dall'altro, tra grammatica 'esplicita' e 'implicita' (quest'ultimo affrontato nel paragrafo 1 a proposito dei procedimenti deduttivi e induttivi). Tali considerazioni sul 'come' gestire la didattica della grammatica si riverberano poi sul 'quando' introdurre la riflessione sulle strutture morfosintattiche all'interno della classe di lingua. Nei seguenti sottoparagrafi affronteremo le suddette questioni contestualizzandole all'interno della nostra proposta sull'uso didattico della canzone.

\section{Insegnamento grammaticale VS riflessione sulla lingua}

L'opposizione tra 'insegnamento grammaticale' e 'riflessione sulla lingua' si inserisce all'interno dell'evoluzione storica degli approcci alla didattica delle lingue esplicitata nel paragrafo 1. Dall'idea di 'insegnamento grammaticale', inteso come trasmissione frontale e verticale delle 
regole morfosintattiche da parte del docente che lo studente deve memorizzare e applicare passivamente, si passa al concetto di 'riflessione sulla lingua', ovvero un procedimento cognitivo in cui lo studente è coinvolto attivamente nell'analisi linguistica del testo della canzone sotto la guida costante dell'insegnante, che ha il compito di aiutarlo nella scoperta delle possibili regolarità e delle eventuali eccezioni morfosintattiche, rendendolo consapevole sia dei modelli linguistici individuati sia delle strategie utilizzate per elaborare ipotesi, fare inferenze e creare generalizzazioni sulle strutture della lingua.

Si tratta di una vera e propria rivoluzione nei ruoli dei partecipanti al processo di acquisizione linguistica, in quanto prevede il ridimensionamento del ruolo del docente a beneficio di un coinvolgimento sempre maggiore dello studente. Quest'ultimo, infatti, smette di essere considerato una tabula rasa su cui il docente deve riversare i contenuti per diventare il protagonista del processo di acquisizione linguistica, sviluppando un'autonomia sempre maggiore e trascendendo l'esclusiva dimensione grammaticale con un miglioramento generale nelle proprie competenze comunicative.

Nella prospettiva della riflessione sulla lingua, Mezzadri considera, infatti, che "l'apprendimento della grammatica [...] diventa non un esercizio fine a se stesso, cioè mirato alla descrizione della lingua, bensì uno strumento dell'uso, indispensabile per capire i meccanismi della lingua e per controllare, migliorandola, la qualità della produzione" (2015, p. 242).

A sostegno della valenza formativa della riflessione grammaticale per la creazione di una forma mentis attivabile sempre e per qualsiasi lingua straniera da parte dello studente, Massimo Vedovelli scrive che la fase di riflessione sulla lingua, definita di "riflessione metalinguistica e metacomunicativa", corrisponde a "un momento ineludibile di ogni equilibrato processo di comunicazione didattica, cioè di comunicazione finalizzata allo sviluppo di una competenza linguistico comunicativa" (2002, p. 144). 
Tempi e spazi della grammatica nell'Unità di Acquisizione

Per poter avviare la riflessione sulla lingua attraverso un procedimento eclettico (inizialmente induttivo e successivamente deduttivo) è necessario avere come punto di partenza un testo corrispondente all' 'input comprensibile' di Krashen, che permetta allo studente di concentrarsi sul significato e non sulla forma, anche grazie alla sua collocazione all'interno dell' 'ordine naturale di acquisizione' nello stadio direttamente seguente a quanto studiato fino a quel momento ('input +1 ') e al non inserimento del 'filtro affettivo' (cfr. Balboni, 2012).

Condizione necessaria per poter avviare la riflessione linguistica è la comprensione dei contenuti della canzone sul piano semantico. Scrive, pertanto, Balboni: "prima si comprende il testo, poi lo si analizza formalmente. Vien prima la pratica del testo e poi quella sul testo, la grammatica. La grammatica è il punto di arrivo, non quello di partenza" (2014, p. 102).

Tali considerazioni rimandano alla più generale strutturazione del1' 'unità di acquisizione' (d'ora in avanti UdA), definita come un blocco minimo di lavoro della durata di una o due ore e percepito come unità di lavoro unitario da parte dello studente, basata a sua volta su fattori neuro e psicolinguistici (cfr. Balboni, 2012). Sulla base dei principi di 'bimodalità' e di 'direzionalità', tali per cui i due emisferi cerebrali cooperano insieme svolgendo funzioni differenti (quello destro è globale, analogico e olistico, mentre quello sinistro è analitico, sequenziale e razionale) e secondo un ordine ben preciso che va dall'emisfero destro a quello sinistro, il testo della canzone deve essere prima compreso globalmente e solo successivamente analizzato nelle singole strutture morfosintattiche.

Sul piano psicolinguistico, secondo la psicodidattica della Gestalt la percezione segue un percorso sequenziale suddiviso nelle tre fasi distinte di globalità, analisi e sintesi. Declinandolo sulla canzone, nella prima fase di globalità si prevede la comprensione generale del testo, con la proposta di attività da svolgere 'prima', 'durante' e 'dopo' l'ascolto ripetuto della canzone mirate a elicitare le preconoscenze, a sfruttare la ridondanza e a formulare delle ipotesi sul significato globale del testo. 
Nella seconda fase di analisi ci si focalizza su obiettivi linguistici specifici, con l'introduzione di attività di verifica delle ipotesi e di analisi dei meccanismi di funzionamento delle strutture - nel nostro caso - morfosintattiche. Ribadiamo come sia fondamentale operare una scelta accurata del testo della canzone che, in quanto materiale autentico, offre potenzialmente molteplici spunti di riflessione ma, allo stesso tempo, può ospitare strutture morfosintattiche di diversi livelli linguistici, sulle quali è consigliabile lavorare solo se presenti in modo significativo nel testo. Nella terza e ultima fase di sintesi si effettua un riepilogo, il più possibile autonomo, con una riflessione esplicita su quanto si è affrontato nel corso dell'UdA attraverso il completamento di mappe e schemi in cui si formalizzano le regole grammaticali scoperte affinché le nuove informazioni possano essere memorizzate stabilmente.

Rispetto alla struttura dell'UdA, i contenuti grammaticali non si affrontano all'interno di una fase specifica, bensì ne permeano più di una a seconda del maggiore o minore riferimento al testo della canzone.

[...] la riflessione grammaticale si colloca a cavallo tra la fase di analisi del testo e quella di sintesi in quanto abbraccia sia attività che presuppongono il ritorno sul testo input dopo il suo ascolto/lettura e la sua comprensione globale e puntuale, sia attività che stimolino gli studenti a riutilizzare autonomamente le strutture apprese per la produzione di nuovi testi. (Diadori, Palermo \& Troncarelli, 2009, p. 148)

Nello specifico, il processo di acquisizione dei contenuti grammaticali si articola in cinque fasi (Balboni, 2014, p. 105):

a. 1'“osservazione" intuitiva e la comprensione dell'input morfosintattico;

b. 1'“elaborazione di ipotesi" sul funzionamento dell'input morfosintattico;

c. la "verifica delle ipotesi" con il reimpiego della regola in attività per verificarne il corretto funzionamento;

d. la "fissazione" delle ipotesi verificate e confermate mediante esercizi ripetuti;

e. la "sistematizzazione" di quanto appreso con la creazione di mappe e schemi di riferimento. 
È evidente come l'iter di acquisizione morfosintattica parta dalla formazione di ipotesi successive all'osservazione delle strutture grammaticali presenti nel testo della canzone (punti a. e b.), per poi staccarsi progressivamente dal testo e riguardare la formulazione di regole ed eventuali eccezioni morfosintattiche all'interno di attività che vanno oltre il testo circoscritto della canzone (punti c. e d.), fino a giungere alla riflessione esplicita e formale sulla lingua (punto e.).

Le tecniche didattiche maggiormente utilizzate nelle prime fasi di osservazione ed elaborazione di ipotesi seguono il procedimento induttivo, anticipano le spiegazioni grammaticali e sono finalizzate alla scoperta di una regola. Sotto la guida dell'insegnante e mediante l'ascolto e la lettura del testo della canzone, gli studenti sono indirizzati al riconoscimento degli elementi morfosintattici attraverso sottolineature, attività di cloze, riempimento di tabelle o schemi per ipotizzare la regola e le eventuali eccezioni.

Per la successiva fase di verifica delle ipotesi si possono proporre degli esercizi di riconoscimento, sostituzione e completamento oppure delle attività meno strutturate basate sulla discussione guidata tra pari o a piccoli gruppi relativamente a quanto ipotizzato nelle fasi precedenti.

Le tecniche didattiche più tradizionali per la fase di fissazione corrispondono ai pattern drill, vale a dire agli esercizi strutturali (come la sostituzione e la trasformazione), finalizzati alla memorizzazione e all'automatizzazione della regola attraverso la ripetizione della sequenza stimolo-risposta-rinforzo. Per ovviare alla ripetitività che li contraddistingue, si possono proporre in chiave ludica nella forma di giochi con i dadi, tris, battaglia navale ecc.

Per l'ultima fase di sistematizzazione si possono utilizzare delle tecniche mirate sia al reimpiego sia alla riflessione esplicita sulle strutture morfosintattiche studiate. Per quanto riguarda le prime, le attività di riutilizzo delle regole possono presentare differenti gradi di creatività (ad esempio, dal completamento di frasi, più guidato e controllato, alla drammatizzazione, più libera e fantasiosa), per permettere agli studenti di esercitarsi in modo sempre più autonomo sulla padronanza dei contenuti grammaticali all'interno di situazioni comunicative scritte e orali. A proposito delle seconde, invece, le tecniche dedicate alla riflessio- 
ne esplicita sulla lingua sono, ad esempio, di tipo insiemistico (come l'inclusione, con la creazione di gruppi omogenei a partire da elementi differenti, l'esclusione, ovvero l'individuazione dell'elemento intruso, e la seriazione, vale a dire il riordino di elementi in disordine secondo un parametro), di manipolazione, riempimento di spazi e di individuazione di forme errate, di combinazione e incastro, di esplicitazione (per rendere evidenti le relazioni tra le componenti del discorso quali i sinonimi, gli iperonimi, gli iponimi, i pronomi e i connettori) $)^{5}$.

\section{CONCLUSIONE}

Sulla base di quanto sostenuto nel presente saggio, se adeguatamente proposta dal docente nei termini di selezione del testo e di predisposizione delle attività, la canzone può costituire una delle risorse da utilizzare a sostegno di una didattica della grammatica fondata sulla centralità del discente, puntando sia sulla motivazione sia sulla considerazione dei suoi interessi e bisogni, senza tralasciare il raggiungimento degli obiettivi didattici prestabiliti.

Tuttavia - e senza che questo risulti contraddittorio rispetto a quanto sostenuto finora - riteniamo fondamentale sottolineare come la canzone sia uno strumento da proporre occasionalmente all'interno del programma didattico. Una UdA sulla canzone si può proporre all'inizio, durante o alla fine della più ampia unità didattica (d'ora in avanti UD), corrispondente a un blocco più complesso di lavoro di durata da sei a dieci ore su un unico tema situazionale e culturale (Balboni, 2012), al fine di:

a. introdurre nuovi argomenti grammaticali per anticipare le strutture morfosintattiche che si affronteranno, contestualizzare l'UD e stimolare la motivazione dei discenti;

b. trattare determinate strutture morfosintattiche all'interno della rete di UdA in cui si sviluppa l'UD;

5 Per un elenco completo delle tecniche didattiche per la didattica della grammatica si vedano, ad esempio, Andorno, Bosc, \& Ribotta, 2003; Balboni, 2014; Diadori, Palermo, \& Troncarelli, 2009; Mezzadri, 2015. 
c. rappresentare delle attività integrative, di rinforzo o di recupero tra le varie attività supplementari presenti alla conclusione dell'UD, sfruttando la ridondanza dei contenuti affrontati con un materiale alternativo e attraverso delle modalità didattiche diverse.

I motivi per cui suggeriamo che l'impiego di tale risorsa didattica non diventi abituale riguardano sia i suoi destinatari sia la natura stessa del materiale.

Da una parte, anche se potenzialmente motivante, se utilizzata troppo frequentemente la canzone può perdere la propria attrattiva, rischiando di non essere più percepita come novità da parte dei discenti. Dall'altra, la canzone è un ottimo materiale per allenare le abilità ricettive e produttive e, nello specifico, per focalizzarsi sulle componenti lessicali e letterarie che la caratterizzano. Si può utilizzare, infatti, per variare la tipologia dei testi da comprendere ed eventualmente per crearne di nuovi, purché di un livello adeguato per gli studenti a cui si pensa di proporla e provvista di una scheda di ascolto altrettanto adatta alle loro competenze linguistico-comunicative (ad esempio, con delle informazioni sui contenuti, sul contesto di composizione e sull'autore e con la spiegazione delle parole chiave). Il suo impiego a fini grammaticali è, dunque, da intendersi a integrazione dei precedenti obiettivi, al fine di poter rendere più piacevole lo studio delle strutture morfosintattiche senza dimenticare l'attenzione ai contenuti semantici e letterari che in origine ha potuto ispirare l'autore del testo.

\section{BIBLIOGRAFIA}

Andorno, C., Bosc, F. \& Ribotta, P. (2003). Grammatica. Insegnarla e impararla. Perugia: Guerra.

Balboni, P.E. (2011). Conoscenza, verità, etica nell'educazione linguistica. Perugia: Guerra.

Balboni, P.E. (2012). Le sfide di Babele. Insegnare le lingue nelle società complesse. Torino: UTET.

Balboni, P.E. (2013). Il ruolo delle emozioni di studente e insegnante nel processo di apprendimento e insegnamento linguistico. EL.LE. Educazione Linguistica - Language Education, 2(1), 7-30. Retrieved from http:// virgo.unive.it/ecf-workflow/upload_pdf/ELLE_4_1.pdf. 
Balboni, P.E. (2014). Didattica dell'italiano come lingua seconda e straniera. Torino: Loescher/Bonacci.

Caon, F. (2006). Pleasure in Language Learning. A Methodological Challenge. Perugia: Guerra.

Caon, F. (2008). Educazione linguistica e differenziazione: gestire eccellenza e difficoltà. Torino: UTET.

Caon, F. (2011). L'italiano parla Mogol: imparare l'italiano attraverso i testi delle sue canzoni. Perugia: Guerra.

Caon, F. \& Serragiotto, G. (ed.). (2012). Tecnologia e didattica delle lingue. Torino: UTET.

Cardona, M. (2009). Musica e apprendimento linguistico. SELM - Scuola e Lingue Moderne, 6, 6-9.

Diadori, P., Palermo, M., \& Troncarelli, D. (2009). Manuale di didattica dell' $i$ taliano L2. Perugia: Guerra.

Fabbro, F. (1996). Il cervello bilingue. Roma: Astrolabio.

Falioni, J.W. (1993). Music as Means To Enhance Cultural Awareness and Literacy in the Foreign Language Classroom. Mid-Atlantic Journal of Foreign Language Pedagogy, 7, 97-108.

Hymes, D. (1972). Models of Interaction of Language and Social Life. In J.J. Gumperz \& D. Hymes (eds.), Directions in Sociolinguistics: the Ethnography of Communication (pp. 35-71). New York: Holt, Rinehart \& Winston.

Krashen, S. (1983). The Din in the Head, Input, and the Second Language Acquisition Device. Foreign Language Annals, 16, 41-4.

Luise, M.C. (2014). Insegnare la grammatica (materiali FILIM - Formazione degli Insegnanti di Lingua Italiana nel Mondo. Università Ca' Foscari Venezia: Laboratorio Itals, Dipartimento di Scienze del Linguaggio). Retrieved from http://venus.unive.it/filim.

Mezzadri, M. (2015). I nuovi ferri del mestiere. Torino: Loescher/Bonacci.

Pasqui, R. (2003). L'utilizzo delle canzoni in glottodidattica. Bollettino Itals. Supplemento alla rivista EL.LE. Retrieved from http://www.itals.it/lutilizzo-della-canzone-glottodidattica.

Santipolo, M. (ed.). (2006). L'italiano. Contesti di insegnamento in Italia e all'estero. Torino: UTET.

Schumann, J.H. (1997). The Neurobiology of Affect in Language. Oxford: Blackwell.

Titone, R. (1987). La dimensione affettiva. In G. Freddi (ed.), Lingue straniere per la scuola elementare (pp. 47-54). Padova: Liviana. 


\section{Vedovelli, M. (2002). Guida all'italiano per stranieri. La prospettiva del Qua- dro comune europeo per le lingue. Roma: Carocci. \\ Wilkins, D.A. (1976). Notional Syllabuses. Oxford: Oxford University Press. \\ Zimmermann, G. (1990). Grammatik im Fremdsprachenunterricht der Er- wachsenenbildung. München: Max Hueber Verlag.}

Riassunto: L'insegnamento della grammatica italiana pone diverse criticità, tra cui la frequente demotivazione degli studenti. Unire le esigenze di insegnamento grammaticale con delle risorse attrattive per i discenti, dunque, si pone come una sfida per il docente di italiano LS.

La canzone può rappresentare un'importante risorsa didattica. Essa, infatti, è molto motivante per gli studenti, è un testo di lingua e cultura estremamente ricco (si pensi alla varietà di registri sociolinguistici o alle figure retoriche presenti) e, grazie alle sue caratteristiche intrinseche (come il ritmo e la melodia), può favorire la memorizzazione efficace del lessico e delle strutture grammaticali.

Per poter sfruttare al meglio le potenzialità della canzone, allora, occorre che il docente di lingua possieda le adeguate conoscenze metodologiche e si doti di un repertorio di tecniche didattiche ampio per presentare i diversi aspetti grammaticali.

Il saggio è diviso in quattro sezioni: nella prima parte si tratta la didattica della grammatica nella prospettiva di una glottodidattica di natura umanistica; nella seconda si considerano gli aspetti critici che la caratterizzano l'insegnamento delle strutture morfosintattiche e si avanzano delle possibili soluzioni, come l'uso didattico della canzone, esplorato nella terza parte; nella quarta e ultima sezione si presentano delle considerazioni metodologiche relative alle modalità e ai tempi di utilizzo della canzone a fini grammaticali.

Parole chiave: educazione linguistica, grammatica, italiano LS, risorsa didattica, canzone 